\title{
Risk Assessment and Prediction of Construction Project Based on 1D-CNN-Attention-BP
}

\author{
Yawen Zhong \\ School of Engineering, Southwest Petroleum University, Nanchong, China \\ Email: arwenzyw@163.com
}

How to cite this paper: Zhong, Y.W. (2021) Risk Assessment and Prediction of Construction Project Based on 1D-CNNAttention-BP. World Journal of Engineering and Technology, 9, 861-876.

https://doi.org/10.4236/wjet.2021.94059

Received: October 11, 2021

Accepted: November 14, 2021

Published: November 17, 202

Copyright $\odot 2021$ by author(s) and Scientific Research Publishing Inc. This work is licensed under the Creative Commons Attribution International License (CC BY 4.0).

http://creativecommons.org/licenses/by/4.0/

\section{(c) (i) Open Access}

\begin{abstract}
In order to solve the problem of low accuracy of construction project duration prediction, this paper proposes a $\mathrm{CNN}$ attention $\mathrm{BP}$ combination model project risk prediction model based on attention mechanism, one-dimensional convolutional neural network (1d-cnn) and BP neural network. Firstly, the literature analysis method is used to select the risk evaluation index value of construction project, and the attention mechanism is used to determine the weight of risk factors on construction period prediction; then, BP neural network is used to predict the project duration, and accuracy, cross entropy loss function and F1 score are selected to comprehensively evaluate the performance of $1 \mathrm{~d}$-cnn-attention-bp combined model. The experimental results show that the duration risk prediction accuracy of the risk prediction model proposed in this paper is more than $90 \%$, which can meet the risk prediction of construction projects with high accuracy.
\end{abstract}

\section{Keywords}

Construction Project Risk, 1D-CNN-Attention-BP, One Dimensional Convolutional Neural Network, Construction Period Forecast, Risk Identification

\section{Introduction}

In the construction project, the contractor strives to win the bid in the form of quotation. In the process of project construction, if the risk factor analysis and evaluation are not carried out objectively, it may lead to wrong decision-making in project bidding and loss of the project. Theoretically, the owner should strive to reasonably disperse the risks when preparing the bidding documents, but few 
owners do so in practice. Therefore, after winning the bid, the contractor of the construction project will bear most of the risks. In order to reduce the risk loss and improve the risk management level, the project risk must be comprehensively analyzed and evaluated before the project construction [1] [2] [3] [4].

With the development of artificial intelligence and big data, neural network has attracted more and more researchers' attention. Due to the strong nonlinear fitting ability of neural network and good effect on the mapping of nonlinear relationships, relevant scholars have combined neural networks with engineering project risk research in recent years and achieved certain results [5] [6] [7]. Reference [8] proposed a risk prediction method of PPP project of underground comprehensive pipe gallery based on PCA-PSO-SVM, which can predict the project risk to a certain extent, but the index weight problem of SVM has not been solved. Literature [9] proposed a project investment deviation, early warning model, based on BP neural network. By defining early warning indicators and combining them with the neural network, the prediction of investment deviation degree of the project is realized. However, BP neural network has the characteristics of many network parameters, difficult to find the global optimal value and serious dependence on samples, which limit the application of traditional $\mathrm{BP}$ neural network in actual engineering project risk prediction. In order to solve the problem that it is difficult to find the global optimal value of BP neural network, literature [10] proposed a project risk prediction method based on PCABP-GA. in order to further improve the prediction accuracy, this paper uses genetic algorithm to optimize BP neural network to avoid the randomness of initial threshold and weight. However, when the selection of evolutionary algebra of the genetic algorithm is large, it will affect the execution efficiency and prediction accuracy of the algorithm.

In order to improve the shortcomings of the neural network prediction model in the above literature, literature [11] proposed a construction project risk prediction model based on EW-FAHP and 1d-cnn, but for a single isolated project, it did not fundamentally change the congenital deficiency of neural network prediction caused by the small amount of project data.

Convolutional neural network can quickly capture the nonlinear relationship of data and alleviate the overfitting problem, which can significantly improve the effect of prediction model [11]. When the vector dimension of input data is too large, it is prone to dimension disaster and reduces the learning efficiency of the model. Attention mechanism is an effective mechanism to obtain good results. Its key is to improve attention to important information and the use attention mechanism to determine the influence weight of risk factors on project duration. By adding the attention mechanism to the convolutional neural network and assigning different probability weights to the middle layer neurons, the neural network model pays more attention to the information with greater impact on the construction period and reduces or even ignores the information with less impact on the construction period [12]. This paper proposes to apply attention to convolutional neural networks $(\mathrm{CNN})$ and add back propagation (BP) to ad- 
just the parameters of the whole combination model. The preprocessed construction period risk factors are used as the input vector to reduce the dimension of neural network. In the CNN hidden layer, the attention mechanism is introduced to the importance of the characteristics of risk factors on the construction period. The feature vector after dimensionality reduction of $\mathrm{CNN}$ layer and attention layer is input into BP neural network to predict the construction period, and the performance of the model is evaluated and analyzed.

\section{Project Risk Theory Research}

\subsection{Project Risk Identification}

Starting from the decision-making stage, various risks affecting the construction project duration and costs will arise as the project progresses. For the construction project management and construction parties, it is necessary to target the entire implementation process of the project with limited resources. Accurately identify the risk factors that have a greater impact on the construction period and costs [13] [14] [15]. For construction project management and construction parties, it is necessary to accurately identify the risk factors that have a greater impact on the construction period and cost for the entire implementation process of the project under the condition of limited resources.

Project risk identification methods mainly include: brainstorming method, literature research method and rough set theory. Each method has its best applicable environment, and suitable identification methods can be selected according to different analysis angles, routes and focuses [16]. Compared with other identification methods, literature research method is not limited by time and space, and can realize risk identification even with a small amount of resources. This method has been widely applied in intelligent algorithms, big data analysis, fault diagnosis, etc. [17] [18].

This paper selects construction projects invested by state-owned assets, controlled by state-owned assets or directly managed by government departments for analysis. Therefore, literature analysis is adopted to identify risk factors and summarize project risk evaluation indexes. Combined with the attributes of construction project risk, the project risk preliminary evaluation index system is obtained.

\subsection{Project Risk Assessment}

In project management, project risk evaluation refers to the process of analyzing, estimating and quantifying the impact of risks on the project. Establishing a scientific and effective risk assessment method is the prerequisite for risk research. The flowchart of the risk assessment process is shown in Figure 1.

\subsection{Construction of Project Risk Evaluation Indicators}

In construction project management, choosing an appropriate risk evaluation index system is the prerequisite for controlling project risks. The selection of risk 


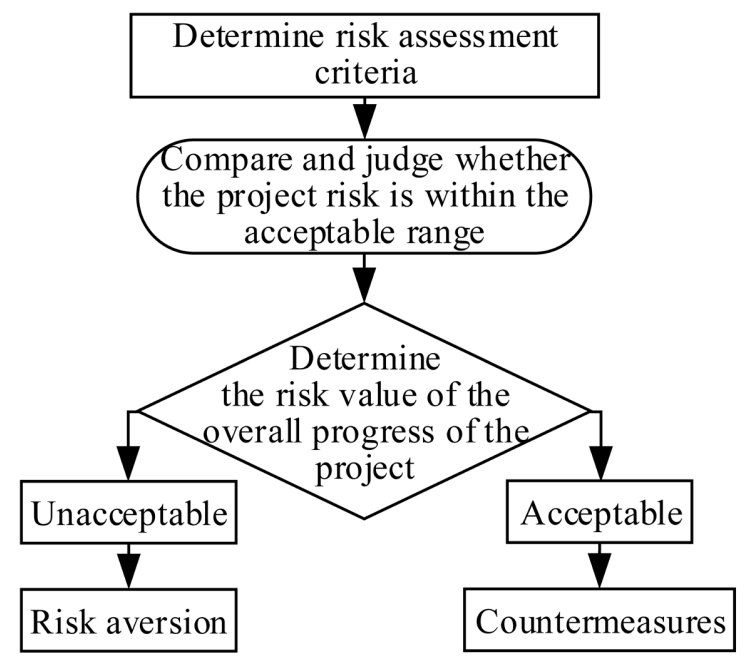

Figure 1. Flow chart of risk assessment process.

assessment indicators should meet the requirements of representativeness, diversity, conciseness and comprehensiveness [19] [20]. The hierarchy of the system determines whether the evaluation index system is scientific and reasonable. Therefore, when constructing the project risk evaluation index system, the evaluation indexes are divided according to the defined grade categories, and finally a multi-level index system is constructed to help risk managers understand the specific conditions of the risks in the project more comprehensively [21] [22]. As is shown in Figure 2, the primary indicators are, and the secondary indicators corresponding to each primary indicator are.

\section{Definition of Construction Project Risk}

In the early stage of decision making, in order to avoid losses, the risks of construction period and cost can be used to evaluate the risk of the whole project before deciding whether to bid. Construction period risk and cost risk can be expressed by Equations (1):

$$
R_{T}=\left|\frac{T_{\Delta}-T_{0}}{T_{\Delta}}\right|
$$

In the formula, $R_{T}$ represent the construction period risk, $T_{\Delta}$ and $T_{0}$ represent the actual construction period and the target construction period respectively.

Table 1 shows the duration risk value of relevant projects of a group in Chengdu established according to formula (1).

\section{Establish Numerical Judgment Matrix}

In order to quantify the decision judgment and form a numerical judgment matrix, the relative importance is given by using the $0.1-0.9$ scale method [23] [24], and the number of index layers is set as, and the initial matrix of Equation (2) is constructed. 


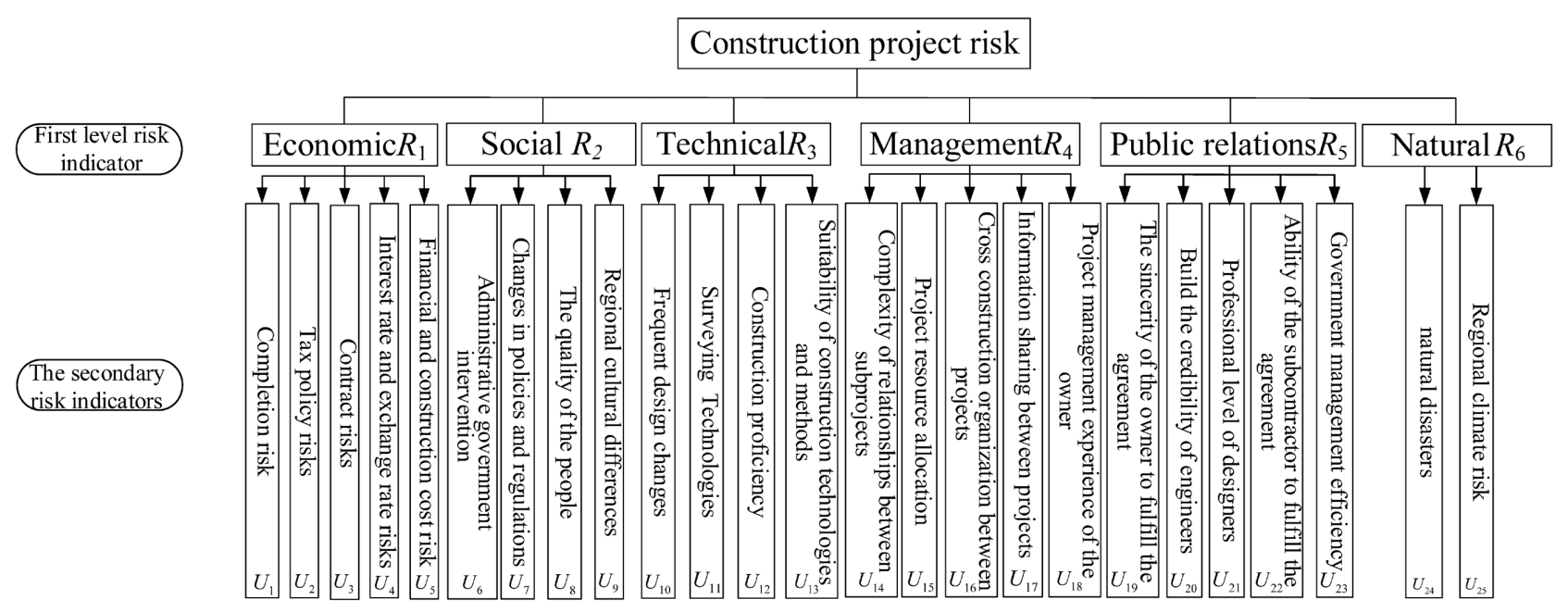

Figure 2. Evaluation index system.

Table 1. Risk factor assessment data of construction project.

\begin{tabular}{cc}
\hline Number & Duration Risk \\
\hline 1 & 0.0659 \\
2 & 0.0842 \\
3 & 0.0682 \\
4 & 0.0734 \\
5 & 0.0649 \\
6 & 0.0685 \\
7 & 0.0651 \\
8 & 0.0523 \\
9 & 0.061 \\
10 & 0.0787 \\
11 & 0.0685 \\
12 & 0.0651 \\
13 & 0.0523 \\
14 & 0.0610 \\
15 & 0.0787 \\
\hline
\end{tabular}

$$
A=\left(a_{i j}\right)_{n \times n}
$$

This paper presents the process of determining the weight of each index of public relations risk, and the weights of other risk factors can be determined sequentially.

The relevant data comes from the data of a Sichuan group's entire construction project in a community in Chengdu. First, use the expert scoring method to fill in the proportional scale table for the public relations risk factors of the construction project, and the following matrix can be obtained: 


$$
A=\left[\begin{array}{lllll}
0.5 & 0.2 & 0.4 & 0.2 & 0.3 \\
0.8 & 0.5 & 0.7 & 0.6 & 0.7 \\
0.6 & 0.3 & 0.5 & 0.3 & 0.4 \\
0.8 & 0.4 & 0.7 & 0.5 & 0.6 \\
0.7 & 0.3 & 0.6 & 0.4 & 0.5
\end{array}\right]
$$

\section{Project Risk Research Model}

\subsection{The Basic Principles of Convolutional Neural Networks}

With the development of neural networks, convolutional neural networks have been applied in more and more fields. They are currently widely used in visual image analysis, natural language processing and recommendation systems [25], but they have not yet been effectively applied in the risk prediction of construction engineering projects.

The CNN (Convolutional Neural Networks) network is an extension of DNN (Deep Neural Networks). It mainly includes input layer, output layer, convolutional layer and pooling layer. A convolution kernel of CNN only extracts one feature, and multiple features are extracted by multiple convolution kernels and then integrated in the fully connected layer [11] [12]. Figure 3 shows the network structure of $1 \mathrm{D}-\mathrm{CNN}$.

The 1D-CNN network structure mainly includes five parts: input layer, convolutional layer, pooling layer, fully connected and output layer. For the input one-dimensional information vector, the vector passes through the convolutional layer and the pooling layer. Finally, the corresponding output is obtained through the fully connected layer.

1) Convolutional layer: Suppose the input signal of the $1 \mathrm{D}-\mathrm{CNN}$ model is $x$, the length is $N$, and the convolution kernel is used to perform convolution operation on the local area of the input signal. The specific convolution operation formula is:

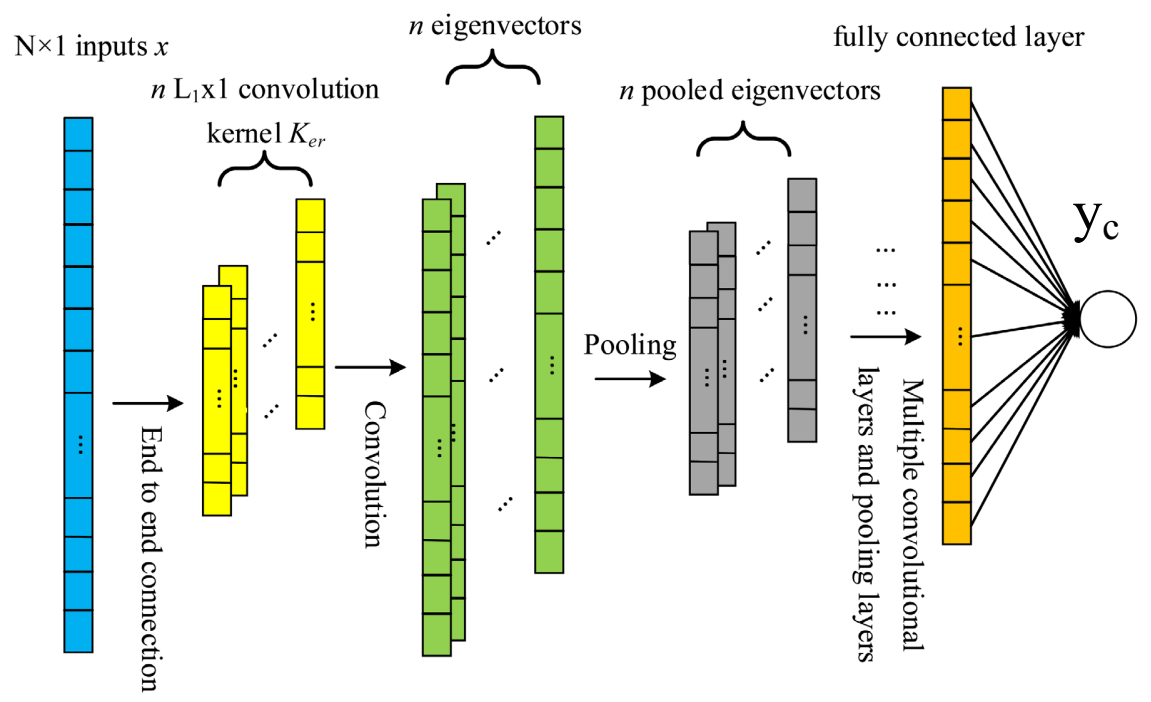

Figure 3. Network Structure of 1D-CNN. 


$$
y_{i}^{k}=\operatorname{Ker}_{L_{1}}^{k} * x_{i}^{k}+b_{i}^{k}\left(i=1,2, \cdots, \frac{N}{s t_{1}}\right)
$$

where: $\operatorname{Ker}_{L_{1}}^{k}$ represents the $k$ th layer convolution kernel whose length is $L_{1}$; * indicates that the convolution operation $x_{i}^{k}$ means the $i$-th input sub-segment (which is the same length with the convolution kernel); $b_{i}^{k}$ represents the offset of the $i$-th convolution output of the $\mathrm{k}$ layer; $y_{i}^{k}$ represents the convolution output of the $k$-th layer; $s t_{1}$ is the convolution step length, where $y^{k}=\left[y_{1}^{k}, y_{2}^{k}, \cdots, y_{N / s t_{1}}^{k}\right]$.

The non-linear processing of the data after the convolution operation is as follows:

$$
s=\max \left(0, y^{k}\right)
$$

In the equation above, $s$ represents the activation function of $y^{k}$. This article uses $\mathrm{ReLu}$, the mainstream activation function in the deep learning world, which can accelerate the model convergence and overcome gradient dispersion.

2) Pooling layer: Pooling layer reduces the calculation amount and reduces the risk of overfitting by reducing the parameters of the neural network. Maximum pooling can be used to obtain position-independent characteristics. The pooling operation is usually the maximum pooling (max-pooling), as shown in Formula (14), the sequence length can be reduced in dimension.

$$
a_{j}=\max _{(j-1) L_{2} \leq t \leq j L_{2}}\left(0, s_{t}^{j}\right)
$$

In the equation: $j=1,2, \cdots, \frac{N}{L_{2} s t_{1}}$, where $s_{t}^{j}$ represents the $t$-th value of the $j$-th pooling segment, $a_{j}$ represents the maximum value of the $j$ th pooling segment; $L_{2}$ represents the length of the pooling segment. The output of the pooling layer is:

$$
a=\left[a_{1}, a_{1}, \cdots, a_{N / L_{2} s t_{1}}\right]
$$

where $a$ is the output vector of the pooling layer.

3) Fully connected layer: The fully connected layer has the same structure as the traditional neural network and is composed of multiple hidden layers. The fully connected layer further abstracts and combines the global timing features, and the output is as follows:

$$
y_{c}=w_{o} a+b_{o}
$$

In the equation, $w_{o}$ and $b_{o}$ are the weight and bias of the fully connected layer, respectively.

\subsection{Principle of Attention Mechanism}

The attention mechanism (Figure 4) is added to the neural network to consider the influence factors of different past states and capture the most important part related to construction period risk. In order to make full use of the information of various risk factors, the $\mathrm{CNN}$ attention $\mathrm{BP}$ combination model proposed in 


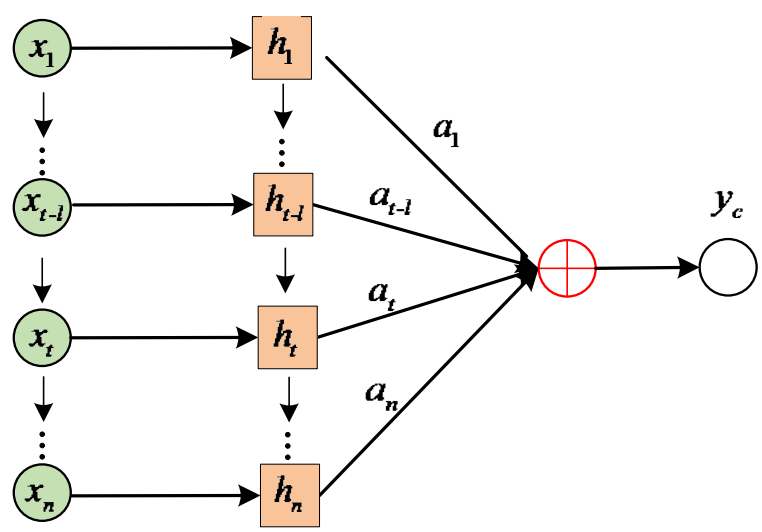

Figure 4. The architecture of attention mechanism.

this paper adds an attention layer to the CNN layer in order to reduce or even ignore the information irrelevant to the construction period and change the attention to important information. The key is to assign different probability weights to neurons, so that the model pays more attention to the large data information affecting classification prediction, so as to improve the prediction accuracy [26].

\subsection{Principle of BP Neural Network}

The learning process consists of signal forward propagation and error back propagation. During forward propagation, the input samples are transmitted from the input layer, processed by each hidden layer, and then transmitted to the output layer. If the actual output of the output layer does not meet the requirements with the expected output, it will turn to the back propagation stage of error. Error back propagation is to back propagate the output error to the input layer by layer through the hidden layer in some form, and allocate the error to all units of each layer, so as to obtain the error signal of each layer unit, which is used as the basis for correcting each unit. The weight adjustment process of each layer of signal forward propagation and error back propagation is carried out repeatedly. The process of continuous weight adjustment is the learning and training process of the network. This process continues until the error of the network output is reduced to an acceptable level or until a predetermined number of learning times.

\subsection{D-CNN-Attention-BP Model}

This paper adopts the combined model structure, which is mainly divided into input layer, CNN layer, attention layer, BP layer and output layer. The process is shown in Figure 5.

The data $X=\left[\begin{array}{cccc}x_{11} & x_{12} & \cdots & x_{1 p} \\ x_{21} & x_{22} & \cdots & x_{2 p} \\ \vdots & \vdots & \ddots & \vdots \\ x_{n 1} & x_{n 1} & \cdots & x_{n p}\end{array}\right]$, related to construction period risk is 


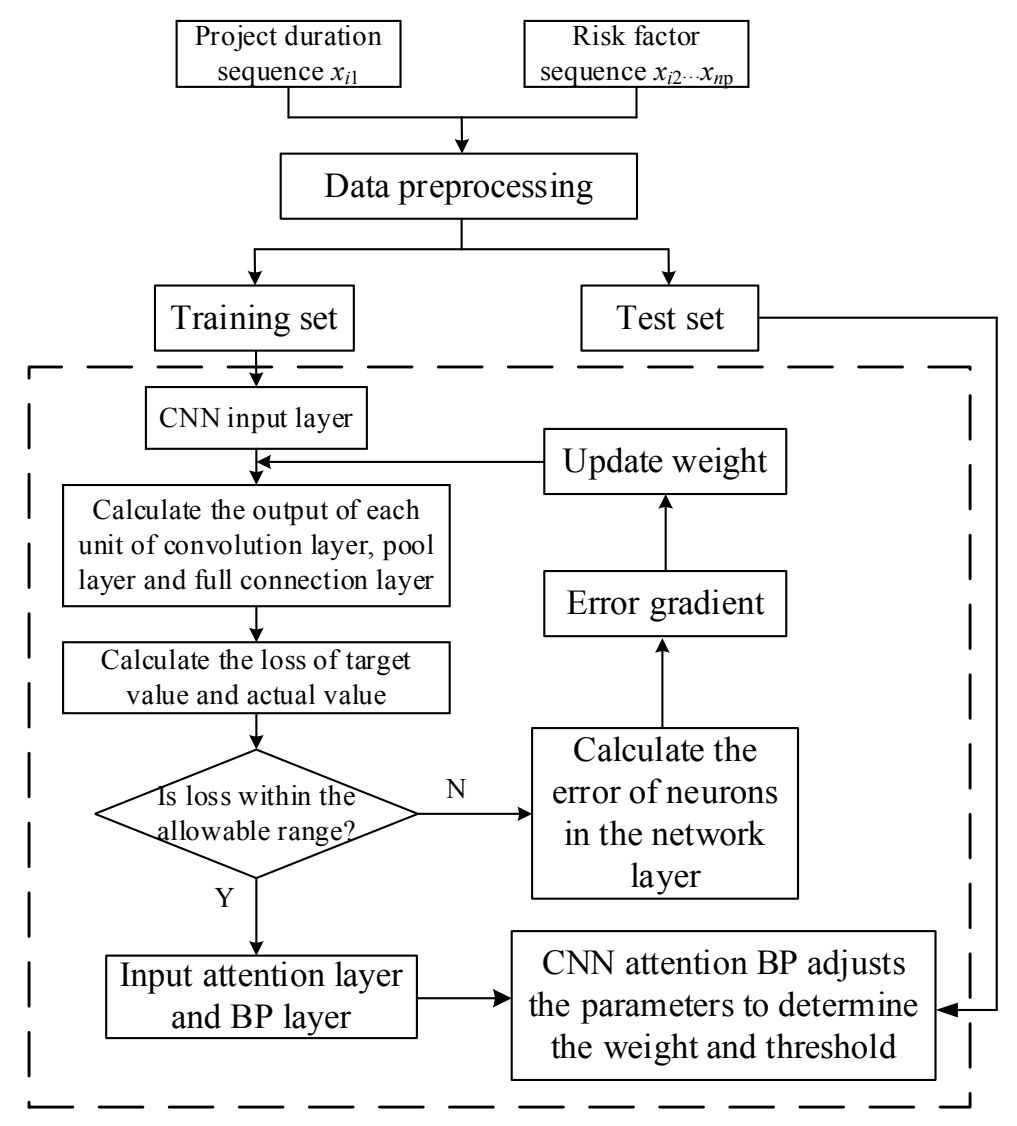

Figure 5. Flow chart of the 1D-CNN-Attention-BPmodel.

used as the input of the combination model. $x_{i 1}$ represents the label value of duration risk, $i=1, \cdots, n ; x_{i 2}, \cdots, x_{n m}$ represents the normalized sequence of the $m$-th impact factor. Firstly, a nonlinear activation function RELU is connected through the convolution layer, and then the commonly used maximum pooling is selected for operation, that is, the maximum value of elements in the pooling layer is extracted. After the convolution layer and pooling layer, the original data is mapped to the hidden layer feature space, and then a full connection layer with sigmoid activation function is built to convert it to output, and finally the output feature vector is obtained [27].

The output eigenvector $y_{c}$ of CNN layer is expressed as:

$$
\begin{gathered}
Z_{1}=f\left(X \otimes W_{1}+b_{1}\right)=\operatorname{ReLU}\left(X \otimes W_{1}+b_{1}\right) \\
F_{1}=\max \left(C_{1}\right)+b_{2} \\
Z_{2}=f\left(F_{1} \otimes W_{2}+b_{3}\right)=\operatorname{ReLU}\left(F_{1} \otimes W_{2}+b_{3}\right) \\
F_{2}=\max \left(Z_{2}\right)+b_{4} \\
Y_{c}=f\left(F_{2} \times W_{3}+b_{5}\right)=\operatorname{sigmoid}\left(Z_{2} \times W_{3}+b_{5}\right)
\end{gathered}
$$

where: $Z_{1}$ and $Z_{2}$ are the convolution outputs of the first layer and the second layer respectively; $F_{1}$ and $F_{2}$ are the output of pool layer 1 and layer 2 respectively; $W_{1}, W_{2}$ and $W_{3}$ are weight matrices; $b_{1}, b_{2}, b_{3}, b_{4}$ and $b_{5}$ are deviation, $\otimes$ 
and $\max ()$ are convolution operation and maximum function, and the length of CNN layer output is $t, Y_{c}=\left[Y_{c 1}, Y_{c 2}, \cdots, Y_{c t}\right]^{\mathrm{T}}$. Then, take the vector $y$ after $\mathrm{CNN}$ hidden layer activation processing as the input of the attention layer, and nonlinearly convert it into a characteristic state matrix representing the output vector $h_{t}$ of the $t$-th sample through CNN layer, as shown in the formula: $e_{t}=u \tanh \left(w_{1} h_{t}+b\right)$.

$u$ and $w_{t}$ are the weight matrix, and $B$ is the offset matrix. Secondly, each variable is given an initial weight, and then normalized through the softmax layer to obtain the attention weight. The calculation method is as follows:

$$
\begin{gathered}
\partial_{t}=\frac{\exp \left(e_{t}\right)}{\sum_{j=1}^{t} \exp \left(e_{j}\right)} \\
s_{t}=\sum_{t=1}^{l} \partial_{t} h_{t}
\end{gathered}
$$

where $\partial_{t}$ is the attention vector and $s_{t}$ is the output of the attention layer at time $t$.

The output vector passing through $\mathrm{CNN}$ and attention layers is recorded as $X_{c}=\left[x_{c 1}, x_{c 2}, \cdots, x_{c l}\right]^{\mathrm{T}}$, which represents the input vector of BP neural network in $\mathrm{L}$ dimension, $Y=\left[y_{1}, y_{2}, \cdots, y_{m}\right]^{\mathrm{T}}$ represents the predicted value, and the forward propagation and backward propagation are carried out after initializing the weight values $W_{p q}$ and $W_{q k}$, the hidden layer threshold $a_{q}$ and the output layer threshold $b_{k}$. In this paper, sigmoid activation function is used at each hidden layer node of $\mathrm{BP}$, and the input of neural node $\mathrm{L}$ is expressed as:

$$
H_{q}=f\left(\sum_{l=1}^{h} W_{p q} x_{l}-a_{q}\right), l=1,2, \cdots, h ; q=1,2, \cdots, s
$$

where $\mathrm{l}$ is the number of hidden layer nodes and $\mathrm{F}$ is the excitation function of hidden layer. The output of the prediction model is obtained through the hidden layer:

$$
O_{k}=\sum_{q=1}^{s} H_{q} W_{q k}-b_{k}, k=1,2, \cdots, r
$$

when $Y_{k}$ is the actual value, there is an error function:

$$
e_{k}=Y_{k}-O_{k}
$$

Finally, the error between the output target vector and the actual value is calculated. If the global error is smaller than the set error value, fix the current weight and threshold parameter values, end the learning, and output the prediction vector $Y=\left[y_{1}, y_{2}, \cdots, y_{f}\right]^{\mathrm{T}}$; If it is not within the error range, the error value is passed back to update the weight and threshold.

\section{Simulation Analysis}

\subsection{Simulation Conditions}

The experimental test platform parameters in this article are Windows 10 Pro- 
fessional 64-bit, processor model (CPU) i7 9850H, main frequency $2.6 \mathrm{GHz}$, and memory (RAM) $2 \times 8$ GB.

The steps of the improved convolutional neural network model are as follows:

Step 1. Divide the normalized construction project duration into training set and test set.

Step 2. Take the processed risk factor data as the input variable and the actual duration value as the output variable, and use the convolution neural network method for training. Set the training cycle (epoch) to 1, and the initial learning rate is 0.01 , batch_Size refers to the number of samples selected for training at one time, which is initially set to 12 to capture the characteristics of the sequence related to the project duration.

Step 3. Introduce attention into CNN hidden layer for training to extract risk factors with strong correlation with construction period.

Step 4. Input the output result of the attention layer into the BP neural network to adjust the parameters of the whole neural network and output the error between the prediction vector and the actual value; If it is not within the error range, return to step 2 to calculate the error gradient again and update the weight; Until it is within the error range, the training ends and the parameter values are fixed. As the accuracy begins to decrease with the increase of epoch, the epoch with the highest accuracy is 9 . Because the over fitting phenomenon occurs when the batch is too large, this paper sets the batch as $32,64,128$ and 256. After preliminary training, when the batch is 128 , the training error is the smallest and the update learning rate is 0.001; See Figure 6 for details. At this time, the training times of $\mathrm{BP}$ is 10 and the learning rate is 0.002 .

Step 5. Use the model after parameter optimization to model the test set data and complete the prediction of construction period.

In this paper, in the training process of combined prediction model, Adam (adaptive motion estimation) [28] algorithm is selected to minimize the objective function and optimize the parameters of neural network. Adam algorithm

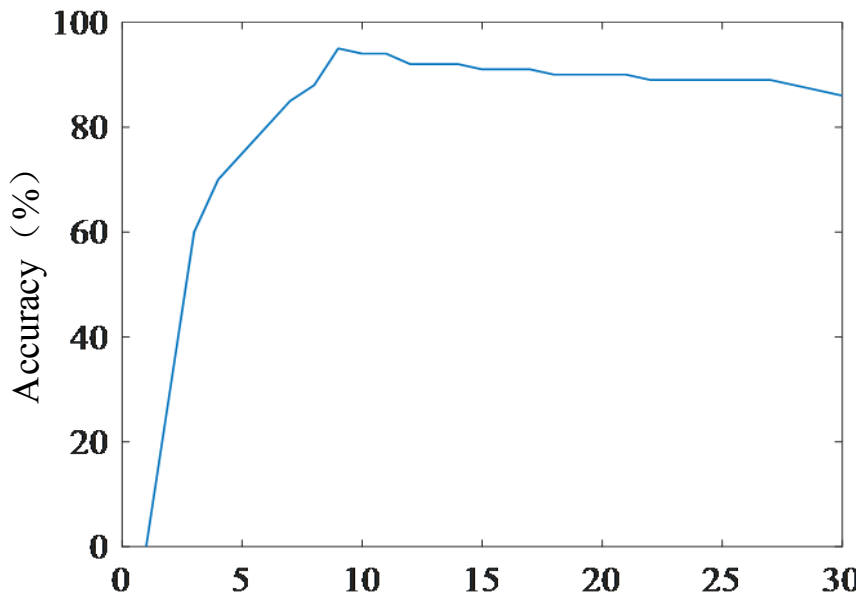

Figure 6. Prediction accuracy of the model with different epochs. 
can iteratively update the weight of neural network based on training data to optimize the output value of loss function. At the same time, the cross entropy loss function is used as the evaluation index, that is:

$$
f_{\text {loss }}=\frac{1}{N} \sum_{l=1}^{f} L_{i}=\frac{1}{N} \sum_{l=1}^{f}-y_{i} \cdot \log \left(p_{i}\right)+\left(1-y_{i}\right) \cdot \log \left(1-p_{i}\right), i=1,2, \cdots, f
$$

where, $f_{\text {loss }}$ is the cross entropy loss function, $y_{i}$ is the expected output of the sample, and $p_{i}$ is the actual output of the sample. Cross entropy is actually the distance between the actual output probability and the expected output probability, that is, the smaller the value of cross entropy, the closer the two probability distributions and the smaller the loss.

Only using the cross entropy loss function can not fully evaluate the performance of the model, so the accuracy (a) that can show the stability of the model and the F1-score ( $\left.F_{1 \text {-score }}\right)$ that can evaluate the generalization ability of the model are selected.

$$
A=\frac{\eta_{T P}+\eta_{T N}}{\eta_{T P}+\eta_{T N}+\eta_{F P}+\eta_{F N}}
$$

where, $\eta_{T P}$ represents the number of samples correctly predicted as having precipitation, $\eta_{T N}$ represents the number of samples correctly predicted as having no precipitation, $\eta_{F P}$ represents the number of samples incorrectly predicted as having precipitation, and $\eta_{F N}$ represents the number of samples incorrectly predicted as having no precipitation.

$$
F_{1 \text {-score }}=\frac{2 P R}{P+R}=\sum_{k=0}^{n} \frac{2 T_{k}^{k}}{\sum_{i=1}^{k-1} P_{i}^{k}+\sum_{j=k}^{n} P_{k}^{j}} \times 100 \%
$$

And:

$$
\begin{gathered}
P=\sum_{k=0}^{n} \frac{T_{k}^{k}}{\sum_{i=1}^{k-1} P_{i}^{k}} \times 100 \% \\
R=\sum_{k=0}^{n} \frac{T_{k}^{k}}{\sum_{j=k}^{n} P_{k}^{j}} \times 100 \% \quad(k=0, \cdots, n)
\end{gathered}
$$

$T_{k}^{k}$ represents the number of samples that are actually positive and predicted to be positive, $P_{i}^{k}$ represents the number of samples that are predicted to be positive by the model, and $P_{k}^{j}$ represents the number of samples that are predicted to be negative by the model.

\subsection{Analysis of Experimental Results}

According to the prediction results in Table 2, the accuracy of 1D-CNN-AttentionBP algorithm in this paper is more than $92 \%$, the loss function is less than 2.6, and $F_{1-s c o r e}$ is about 0.7 . The simulation results further verify the reliability and accuracy of the proposed algorithm. 
Table 2. Relationship between evaluation set and corresponding quantity value.

\begin{tabular}{cccccc}
\hline Number & actual value & Estimate & Accuracy & loss function & $\mathrm{F}_{1}$-score \\
\hline 1 & 0.0659 & 0.0610 & $93 \%$ & 0.232 & 0.732 \\
2 & 0.0842 & 0.0801 & $95 \%$ & 0.201 & 0.761 \\
3 & 0.0682 & 0.0740 & $92 \%$ & 0.255 & 0.693 \\
4 & 0.0734 & 0.0786 & $93 \%$ & 0.247 & 0.702 \\
5 & 0.0649 & 0.0596 & $92 \%$ & 0.254 & 0.691 \\
6 & 0.0685 & 0.0631 & $92 \%$ & 0.254 & 0.712 \\
7 & 0.0651 & 0.0692 & $94 \%$ & 0.213 & 0.753 \\
8 & 0.0523 & 0.0563 & $93 \%$ & 0.241 & 0.742 \\
9 & 0.0610 & 0.0579 & $95 \%$ & 0.206 & 0.771 \\
10 & 0.0787 & 0.0824 & $96 \%$ & 0.191 & 0.787 \\
\hline
\end{tabular}

Table 3. Comparison of prediction results of various prediction models.

\begin{tabular}{cc}
\hline \multirow{2}{*}{ prediction model } & Predicted project duration/error \\
\cline { 2 - 2 } 1D-CNN-Attention-BP & 973 days \\
BP & $914 / 6.1 \%$ \\
SVM & $1076 / 10.6 \%$ \\
ELM & $825 / 15.2 \%$ \\
\hline
\end{tabular}

\subsection{Comparative Analysis of Prediction Model Performance}

In order to verify the effect of the $1 \mathrm{D}-\mathrm{CNN}$-Attention-BP risk prediction model proposed in this paper on risk prediction, BP (back propagation), SVM (support vector machine) and elm (extreme learning machine) networks are selected to predict and compare the construction project duration risk and cost risk discussed in this paper. 10 groups of data with item No. 6 - 15 in Table 1 are selected for risk prediction, and compared with the real value. Table 3 shows the relevant information of a group company in Sichuan in the construction of a residential community in Chengdu and the prediction results of each prediction model.

It can be seen from Table 3 that the 1D-CNN-Attention-BP risk prediction curve has the smallest error and the closest curve to the real value, indicating that the $1 \mathrm{D}-\mathrm{CNN}$-Attention-BP risk prediction model proposed in this paper has better prediction accuracy and effect than other risk prediction models.

\section{Conclusion}

Due to the uncertainty of early construction period, this paper proposes a combined model based on 1d-cnn-attention-bp to decompose the complex construction period prediction task. Firstly, the convolution neural network is used to effectively learn the risk factor information related to the construction period for 
feature extraction. Then the attention mechanism is used to distribute the weight of important information. Finally, BP is used to adjust the parameters of the whole network to achieve optimal prediction results. The results show that the accuracy of the CNN attention BP combined model proposed in this paper can reach about $90 \%$, which is greatly improved compared with the traditional prediction model. The new combined model has better stability and accuracy in construction period prediction and has better applicability.

\section{Acknowledgements}

This research was supported by Nanchong Science and Technology Bureau Project (No.19SXHZ0040).

\section{Conflicts of Interest}

The author declares no conflicts of interest regarding the publication of this paper.

\section{References}

[1] Wang, Q.K. and Wang, Y.H. (2018) ANP-Based Research on the Strategic Risk Assessment for Multi Project Management in Prefabricated Buildings. Journal of $\mathrm{Wu}^{-}$ han University of Technology, 40, 76-79.

[2] Xiao, Q.D., Zhao, Z.N. and Liu, L.C. (2021) Research on Construction Risk Management of Subway Project Based on Bayesian Network. Journal of Xinyang Normal University (Natural Science Edition). https://kns.cnki.net/kcms/detail/41.1107.N.20201207.1007.002.html

[3] Chen, K., Chen, X., Wei, X., et al. (2019) Bayesian Network-Based Risk Analysis on the Blowout of the Shale Gas Wells. Journal of Safety and Environment, 19, 226-241.

[4] Stanković, M., Gladović, P. and Popović, V. (2019) Determining the Importance of the Criteria of Traffic Accessibility Using Fuzzy AHP and Rough AHP Method. Decision Making: Applications in Management and Engineering, 2, 86-104.

[5] Badi, I., Abdulshahed, A., Shetwan, A. and Eltayeb, W. (2019) Evaluation of Solid Waste Treatment Methods in Libya by Using the Analytic Hierarchy Process. Decision Making: Applications in Management and Engineering, 2, 19-35.

[6] Petrovic, I. and Kankaras, M. (2020) A Hybridized IT2FS-DEMATEL-AHP-TOPSIS Multicriteria Decision Making Approach: Case Study of Selection and Evaluation of Criteria for Determination of Air Traffic Control Radar Position. Decision Making: Applications in Management and Engineering, 3, 146-164. https://doi.org/10.31181/dmame2003134p

[7] Ehsan, E., Nima, K., Ezutah, U.O., Grosse, E.H. and Schwindl, K. (2017) Applying Fuzzy Multi-Objective Linear Programming to a Project Management Decision with Nonlinear Fuzzy Membership Functions. Neural Computing and Applications, 28, 2193-2206. https://doi.org/10.1007/s00521-015-2160-0

[8] Jin, J., Li, Z.H., Zhu, L., et al. (2019) Application of BP Neural Network in Risk Evaluation of Railway Construction. Journal of Railway Engineering Society, 3, 103-109.

[9] Lu, X.Q., Huang, Y.J. and Wang, X. (2017) Intelligent Evaluation Model Based on 
PCA-RBF Neural Network Applied to Risk Assessment of PPP Projects. Science and Technology Management Research, 14, 59-63.

[10] Zhou, J., Koopialipoor, M., Li, E. and Armaghani, D.J. (2020) Prediction of Rockburst Risk in Underground Projects Developing a Neuro-Bee Intelligent System. Bulletin of Engineering Geology and the Environment, 79, 4265-4279. https://doi.org/10.1007/s10064-020-01788-w

[11] Gao, C.L., Li, S.C., Wang, J., Li, L. and Lin, P. (2018) The Risk Assessment of Tunnels Based on Grey Correlation and Entropy Weight Method. Geotechnical and Geological Engineering, 36, 1621-1631. https://doi.org/10.1007/s10706-017-0415-5

[12] Khorram, S. (2020) Correction to: A Novel Approach for Ports' Container Terminals' Risk Management Based on Formal Safety assessment: FAHP-Entropy Measure-VIKOR Model. Natural Hazards, 103, 1709.

https://doi.org/10.1007/s11069-020-04160-Z

[13] Guo, Y.H., Shi, Y.C. and Xu, Y.J. (2017) Evaluation of Bridge Construction Quality based on Improved FAHP Evaluation Method. Journal of Civil Engineering and Management, 34, 44-48.

[14] Zhou, F.Y., Jin, L.P. and Dong, J. (2017) A Review of Convolutional Neural Networks. Journal of Computers, 40, 1229-1251.

[15] Morgunova, E.P. (2018) Investment Project Risk Identification and Evaluation. The International Science and Technology Conference "FarEastCon", Vol. 138, Vladivostok, 2-4 October 2018, 186-201. https://doi.org/10.1007/978-3-030-15577-3_19

[16] Sanghera, P. (2019) Project Risk Management. In: CAPM in Depth, Apress, Berkeley, 417-468. https://doi.org/10.1007/978-1-4842-3664-2_11

[17] Schatteman, D., Herroelen, W., Stijn, V.D.V. and Boone, A. (2008) Methodology for Integrated Risk Management and Proactive Scheduling of Construction Projects. Journal of Construction Engineering and Management, 134, 885-893. https://doi.org/10.1061/(ASCE)0733-9364(2008)134:11(885)

[18] Mulgan, G. (2018) Artificial Intelligence and Collective Intelligence: The Emergence of a New Field. AI \& SOCIETY, 33, 631-632. https://doi.org/10.1007/s00146-018-0861-5

[19] Anysz, H. and Buczkowski, B. (2019) The Association Analysis for Risk Evaluation of Significant Delay Occurrence in the Completion Date of Construction Project. International Journal of Environmental Science and Technology, 16, 5369-5374. https://doi.org/10.1007/s13762-018-1892-7

[20] Yu, X.J. and Peng, Y.Y. (2017) The Application and Challenges of Artificial Intelligence in the Field of Financial Risk Management. Southern Finance, 9, 70-74.

[21] Wu, Q., Gao, S.H. and Zhou, T. (2011) Comprehensive Evaluation of Construction Project Schedule Control. Journal of Xi an University of Science and Technology, 4 , 412-419.

[22] Li, S.C. and Wu, J. (2019) A Multi-Factor Comprehensive Risk Assessment Method of Karst Tunnels and Its Engineering Application. Bulletin of Engineering Geology and the Environment, 78, 1761-1776. https://doi.org/10.1007/s10064-017-1214-1

[23] Zhong, Y.W. (2018) Study on Schedule Risk of Project Group based on Rough Set Theory. Xihua University, Chengdu.

[24] Li, L., Li, S.Y., He, W.J., et al. (2020) Emergency Capability Evaluation of Construction Projects Based on EM and FAHP. Journal of Xi an University of Science and Technology, 4, 572-579.

[25] Yu, C., Luo, B., Wang, D.G., et al. (2020) Evaluation of Cultivated Land Consolida- 
tion Potential Based on Improved FAHP-Entropy Weighting Method. China Agricultural Resources and Regional Planning, 41, 15-24.

[26] Wu, X., Hua, Y., Guan, Y., et al. (2021) Application of CNN-Attention-BP to Forecast of Whether Precipitation. Journal of Nanjing University of Information Science \& Technology (Natural Science Edition).

https://kns.cnki.net/kcms/detail/32.1801.N.20210923.1519.002.html

[27] Liang, Z.Y., Du, J.P. and Li, C.Y. (2020) Abstractive Social Media Text Summarization Using Selective Reinforced Seq2Seqattention Model. Neurocomputing, 410, 432-440. https://doi.org/10.1016/j.neucom.2020.04.137

[28] Xie, H. (2014) Prediction of Driving Condition for Plug-in Hybrid Electric Vehicles. Master's Thesis, Chongqing University, Chongqing. 